\title{
Evaluación de Varios Niveles de Fertilización en Aplicación Edáfica y en Fertiriego en el Cultivo de Plátano (Musa $A A B$ Simmonds). El Carmen. Manabí.
}

Diego Vaca Sotelo, Marcelo Calvache ${ }_{2}$

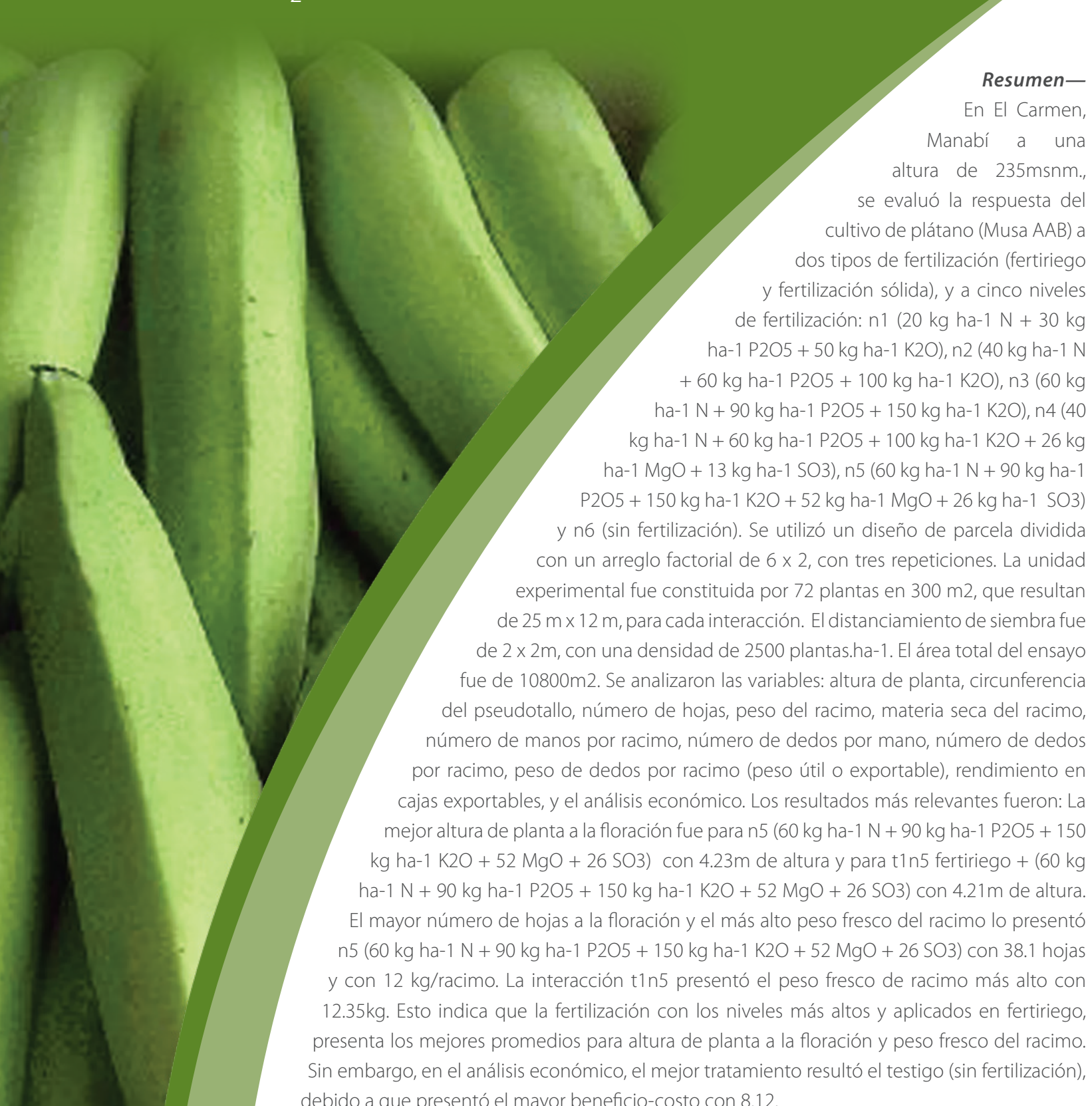

Palabras clave- Aplicación Edáfica, Cultivo, Fertilización.

1 Diego Vaca, Egresado Maestría en Nutrición Vegetal, 099663623.

2 Marcelo Calvache, 2. PhD. Profesor de la Universidad Tecnológica Equinoccial, Maestría en Nutrición Vegetal (Director de Tesis). 


\section{Introducción}

La producción platanera en el Ecuador tiene importancia significativa, por el consumo generalizado de este producto, que conjuntamente con el arroz y la yuca, constituyen alimentos básicos en la población del litoral, especialmente en el área campesina, Orellana (2002). Este cultivo ha sido ancestral en el país principalmente para el consumo interno. La presión de la demanda étnica en países como Estados Unidos y Europa, han estimulado la producción de plátano de buena calidad para la exportación. El plátano barraganete (Musa paradisiaca L), es después del plátano dominico, el de mayor producción y consumo más extendido en el litoral ecuatoriano, en un nivel estimado del 35\%. Es además, la variedad destinada a la exportación, por ser la preferida de la población de origen caribeño que reside en EEUU, Carranza (1999).

A pesar de la importancia que tiene el cultivo de plátano (Musa AAB) en el Ecuador, y a pesar de ser el motor económico en la principal zona platanera del país, es decir en el cantón El Carmen, la gran mayoría de los productores manejan el plátano como un cultivo perenne, con escaso manejo técnico. Existen pocos agricultores que aplican fertilizantes, pero lo hacen de manera empírica, debido principalmente a que no tienen los fundamentos técnicos para desarrollar esta actividad adecuadamente. Existen plataneras con más de 40 años sin ser renovadas, y con productividades muy bajas que no llegan a las 4 tm.ha-1.año-1. Se suma a esto la temporada de verano, que cada año presenta menos precipitaciones, debido a ello, el cultivo entra en estrés hídrico, lo que reduce al menos el 50\% del rendimiento. Debido a ello, se ha empezado a instalar sistemas de riego en el cultivo de plátano en El Carmen, con el fin de mejorar la producción del cultivo.

Tomando en consideración estos aspectos se creyó conveniente realizar una investigación que se enfoque sobre la respuesta del cultivo de plátano al fertiriego tomando en cuenta los siguientes objetivos:

- Determinar la forma de aplicación fertilización química y la cantidad de estos, que ofrezca los mayores rendimientos para la localidad en estudio y realizar el análisis económico de los tratamientos.

\section{Materiales y Métodos}

La investigación se desarrolló en la finca "Santa Marianita", ubicada en el Recinto "San Agustín", cantón "El Carmen", provincia de Manabí, a una altitud de 235 m.s.n.m., la temperatura promedio es de $24.3^{\circ} \mathrm{C}$, precipitación promedio anual $2846 \mathrm{~mm}$ y la humedad relativa del 86.2\%. La textura es franco-limosa.
En la investigación se evaluó dos formas de fertilización química en plátano barraganete, con cinco niveles de fertilización. Los tipos de fertilización fueron: t1 (sólida edáfica) y t2 (fertiriego). Y los niveles fueron: n1 (20 kg ha-1 N + 30 kg ha-1 P2O5 + 50 kg ha-1 K2O), n2 (40 kg ha-1 N + 60 kg ha-1 P2O5 + 100 kg ha-1 K2O), n3 (60 kg ha-1 N + 90 kg ha-1 P2O5 + 150 kg ha-1 K2O), n4 (40 kg ha-1 N + 60 kg ha-1 P2O5 + 100 kg ha-1 K2O + 26 kg ha-1 MgO + 13 kg ha-1 SO3), n5 (60 kg ha-1 N + 90 kg ha-1 P2O5 + 150 kg ha-1 K2O + 52 kg ha-1 MgO + 26 kg ha-1 SO3) y n6 (sin fertilización). Se utilizó un diseño de parcela dividida $6 \times 2$, con tres repeticiones, en la parcela grande se ubicó, tipos de fertilización y en la subparcela niveles de fertilización.

La unidad experimental estuvo constituida por 72 plantas en $300 \mathrm{~m} 2$, que resultan de $25 \mathrm{~m} \times 12 \mathrm{~m}$, para cada interacción. El distanciamiento de siembra fue de $2 \times 2 \mathrm{~m}$, con una densidad de 2500 plantas.ha-1. El área total del ensayo fue de $10800 \mathrm{~m} 2$.

Las variables evaluadas fueron: altura de planta, circunferencia de pseudotallo, número de hojas a la floración, peso del racimo, rendimiento, materia seca por racimo y análisis económico.

El manejo del experimento consistió en: realizar el análisis de suelo, se instaló el sistema de riego por aspersión subfoliar, se diseñó el trazado del ensayo y la fertilización edáfica se aplicó de acuerdo a niveles de cada una de las interacciones, con la siguiente distribución:

\begin{tabular}{|l|c|l|c|c|c|}
\hline Elemento & Transplante & 2 meses & 4 meses & 6 meses & 9 meses \\
$\mathrm{N}$ & $20 \%$ & $20 \%$ & $20 \%$ & $20 \%$ & $20 \%$ \\
$\mathrm{P}$ & $25 \%$ & $25 \%$ & & $50 \%$ & \\
$\mathrm{~K}$ & $20 \%$ & $20 \%$ & $20 \%$ & $20 \%$ & $20 \%$ \\
$\mathrm{Mg}$ & $30 \%$ & & $40 \%$ & & $30 \%$ \\
$\mathrm{~S}$ & $30 \%$ & & $40 \%$ & & $30 \%$ \\
\hline
\end{tabular}

El fertiriego se hizo semanalmente, hasta los nueve meses de plantación. Las labores de manejo del cultivo como: deshoje, chapia y deschante, se realizaron periódicamente de acuerdo al cronograma de actividades. El enfunde se realizó a partir de la floración. Se realizó una aplicación foliar complementaria de Ca y B, debido a que se observó síntomas de deficiencia de estos elementos en todo el ensayo. No se utilizó ningún tipo de pesticidas químicos. La cosecha se realizó cuando los racimos tenían de ocho a diez semanas a partir del enfunde, para que cumplan con las exigencias en calibración que establecen las empresas exportadoras de plátano (de 42 a $58 \mathrm{~mm}$ de diámetro). 


\section{Resultados y Discusión}

\section{Altura de planta}

Al realizar el análisis estadístico para la variable altura de planta a las 20 semanas, Cuadro 1, no se observa diferencias estadísticas para ninguno de los factores en estudio. El coeficiente de variación fue de 11.4\% para tipo de fertilización y de $12.39 \%$ para niveles de fertilización. El promedio general para esta variable fue de $126.2 \mathrm{~cm}$ de altura.

En el análisis de varianza a las 30 semanas Cuadro 1 , se observa diferencias altamente significativas para niveles de fertilización y significativas para la interacción T x N, y ninguna significación estadística para tipo de fertilización. El coeficiente de variación fue de 6.15 para tipos de fertilización y de 7.17\% para niveles de fertilización, aceptable para este tipo de experimentos. El promedio general del experimento fue de $208.34 \mathrm{~cm}$.

Tukey al 5\% para altura a las 30 semanas, Cuadro 2, establece dos rangos de significancia para niveles de fertilización, ubicándose en el primer rango los niveles n5 (60 kg ha-1 N + 90 kg ha-1 P2O5 + 150 kg ha-1 K2O + $52 \mathrm{MgO}+$ 26 SO3), con $223.77 \mathrm{~cm}$ de altura, n4 (40 kg ha-1 N + $60 \mathrm{~kg}$ ha-1 $\mathrm{P} 2 \mathrm{O} 5+100 \mathrm{~kg}$ ha-1 $\mathrm{K} 2 \mathrm{O}+26 \mathrm{MgO}+13 \mathrm{SO} 3) \mathrm{con} 222.67 \mathrm{~cm}$ y n3 (60 kg ha-1 N + 90 kg ha-1 P2O5 + 150 kg ha-1 K2O) con 222.13, en el último lugar está el testigo (sin fertilización) con 186.2 cm. En la interacción Tx N, Cuadro 2, aparecen tres rangos de significancia, ubicándose en el primer rango t1n4 (fertiriego x 40 kg ha-1 N + 60 kg ha-1 P2O5 + 100 kg ha-1 K2O + 26 MgO + 13 SO3) con 233.47 cm, y t2n5 (fertilización edáfica x 60 kg ha-1 N + 90 kg ha-1 P2O5 + 150 kg ha-1 K2O + 52 MgO + 26 SO3), con $223.20 \mathrm{~cm}$ de altura, en el último lugar se encuentra el testigo con $181.87 \mathrm{~cm}$.

En el análisis de varianza a las 40 semanas Cuadro 1, se observa diferencias significativas para niveles de fertilización y altamente significativas para la interacción $T$ x N, y ninguna significación estadística para tipo de fertilización. El coeficiente de variación fue de $2.57 \%$ para tipos de fertilización y de 1.79\% para niveles de fertilización, muy bueno para este tipo de investigación. El promedio general del experimento fue de $415.44 \mathrm{~cm}$.

Tukey al 5\% para altura a las 40 semanas, Cuadro 2 , establece tres rangos de significancia para niveles de fertilización, ubicándose en el primer rango los niveles n5 (60 kg ha-1 N + 90 kg ha-1 P2O5 + 150 kg ha-1 K2O + 52 MgO + 26 SO3), con $423.25 \mathrm{~cm}$ de altura, en el último lugar está n1 (20 kg ha-1 N + 30 kg ha-1 P2O5 + 50 kg ha-1 K2O), con 407.6 $\mathrm{cm}$. En la interacción $\mathrm{T} \times \mathrm{N}$, Cuadro 2, aparecen tres rangos de significancia, ubicándose en el primer rango t1n5 (fertiriego $\mathrm{x}$ 60 kg ha-1 N + 90 kg ha-1 P2O5 + 150 kg ha-1 K2O + 52 MgO + 26 SO3), con $429.57 \mathrm{~cm}$ y en el último lugar se encuentra la interacción t1n1 (fertiriego con 20 kg ha-1 N + 30 kg ha-1 P2O5 +50 kg ha-1 K2O), con $401.97 \mathrm{~cm}$ de altura

Cuadro 1. Adeva para altura de planta a las 20, 30 y 40 semanas del transplante en el estudio de tipos y niveles de fertilización en plátano (Mussa AAB). El Carmen. Manabí. 2007.

\begin{tabular}{|c|c|c|c|c|}
\hline F de V & GL & & drados Medios & \\
\hline & & 20 semanas & 30 semanas & 40 semanas \\
\hline Total & 35 & $1057.26 \mathrm{~ns}$ & $667.64 n s$ & 72.90ns \\
\hline Tipo de Fertilizac. & 1 & $635.04 \mathrm{~ns}$ & $108.51 \mathrm{~ns}$ & $67.24 n s$ \\
\hline Error (a) & 2 & 207.13 & 164.00 & 114.28 \\
\hline Niveles de Fertil. & 5 & $373.43 n s$ & $1644.86^{* *}$ & $193.42^{*}$ \\
\hline t vs. $n 1, n 2, n 3, n 4, n 5$ & 1 & $13.14 \mathrm{~ns}$ & $98.00 \mathrm{~ns}$ & $5.35 \mathrm{~ns}$ \\
\hline $\mathrm{n} 5$ vs. $\mathrm{n} 1, \mathrm{n} 2, \mathrm{n} 3, \mathrm{n} 4$ & 1 & $1.22 \mathrm{~ns}$ & $25.22 \mathrm{~ns}$ & $9.57 \mathrm{~ns}$ \\
\hline n4 vs. n1,n2,n3 & 1 & $0.18 \mathrm{~ns}$ & $35.58 \mathrm{~ns}$ & $0.23 \mathrm{~ns}$ \\
\hline $\mathrm{n} 2$ vs. n3 & 1 & $36.69 \mathrm{~ns}$ & $62.87 \mathrm{~ns}$ & $0.02 \mathrm{~ns}$ \\
\hline n4 vs. n5 & 1 & $0.38 \mathrm{~ns}$ & $0.10 \mathrm{~ns}$ & $4.62 \mathrm{~ns}$ \\
\hline$T \times N$ & 5 & $300.23 \mathrm{~ns}$ & $616.63^{*}$ & $245.24^{* *}$ \\
\hline Error (b) & 20 & 244.60 & 223.24 & 55.19 \\
\hline
\end{tabular}


Cuadro 2. Promedios y pruebas de significancia para altura de planta en el estudio de tipos y niveles de fertilización en plátano (Mussa AAB). El Carmen. Manabí. 2007.

\begin{tabular}{|l|l|l|l|l|}
\hline \multirow{2}{*}{ Factores } & & \multicolumn{3}{|c|}{ Promedios (cm/planta) } \\
Tipos de Fertilizante & Significado & 20 semanas & 30 semanas & 40 semanas \\
T1 & Fertiriego & 130.42 & 206.60 & 416.81 \\
T2 & Fertilic. sólida & 122.02 & 210.07 & 414.07 \\
Niveles de Fertizante & & & & \\
N1 & $20-30-50$ & 127.52 & $200.58 \mathrm{~b}$ & $407.60 \mathrm{c}$ \\
N2 & $40-60-100$ & 118.88 & $194.67 \mathrm{~b}$ & $417.60 \mathrm{abc}$ \\
N3 & $60-90-150$ & 139.87 & $222.13 \mathrm{a}$ & $418.12 \mathrm{ab}$ \\
N4 & $40-60-100-26-13$ & 127.57 & $222.67 \mathrm{a}$ & $415.80 \mathrm{abc}$ \\
N5 & $60-90-150-52-26$ & 125.43 & $223.77 \mathrm{a}$ & $423.25 \mathrm{a}$ \\
N6 & testigo & 118.03 & $186.20 \mathrm{~b}$ & $410.27 \mathrm{bc}$ \\
NxT & & & & \\
t1n1 & & 140.60 & $216.30 \mathrm{abc}$ & $401.97 \mathrm{c}$ \\
t1n2 & & 130.03 & $189.07 \mathrm{abc}$ & $428.70 \mathrm{ab}$ \\
t1n3 & & 14160 & $216.73 \mathrm{abc}$ & $418.40 \mathrm{abc}$ \\
t1n4 & & 124.73 & $233.47 \mathrm{a}$ & $416.43 \mathrm{abc}$ \\
t1n5 & & 131.33 & $214.33 \mathrm{abc}$ & $4209.57 \mathrm{a}$ \\
testigo & & 114.20 & $190.53 \mathrm{abc}$ & $405.77 \mathrm{c}$ \\
t2n1 & & 114.43 & $184.87 \mathrm{bc}$ & $413.23 \mathrm{abc}$ \\
t2n2 & & 138.13 & $227.53 \mathrm{ab}$ & $417.83 \mathrm{abc}$ \\
t2n3 & & 130.40 & $211.87 \mathrm{abc}$ & $415.17 \mathrm{abc}$ \\
t2n4 & & 119.53 & $233.20 \mathrm{a}$ & $416.93 \mathrm{abc}$ \\
t2n5 & & 121.57 & $181.87 \mathrm{c}$ & $414.77 \mathrm{abc}$ \\
testigo & & & &
\end{tabular}

Número de Hojas

Al realizar el análisis estadístico para la variable número de hojas a las 20 semanas, Cuadro 3, no se observa diferencias estadísticas para ninguno de los factores en estudio. El coeficiente de variación fue de $10.45 \%$ para tipo de fertilización y de $4.28 \%$ para niveles de fertilización. El promedio general para esta variable fue de 16.76 hojas a las 20 semanas.

En el análisis de varianza a las 30 semanas Cuadro 3, se observa diferencia significativa para niveles de fertilización y ninguna significación estadística para tipo de fertilización y la interacción T X N. El coeficiente de variación fue de 5.81\% para tipos de fertilización y de $2.95 \%$ para niveles de fertilización, aceptable para este tipo de experimentos. El promedio general del experimento fue de 23.78 hojas a las 30 semanas.
Tukey al 5\% para número de hojas a las 30 semanas, Cuadro 4, establece dos rangos de significancia para niveles de fertilización, ubicándose en el rango "a" los niveles n3 con 24.33 hojas, n5, n4 y n1. En último lugar en el rango "b" está n2 (40 kg ha-1 N+60 kg ha-1 P2O5 + 100 kg ha-1 K2O), con 22.87 hojas.

En el análisis de varianza a las 40 semanas Cuadro 3, se observa diferencias significativas para niveles de fertilización y ninguna significación estadística para tipo de fertilización y la interacción T x N. El coeficiente de variación fue de 3.14\% para tipos de fertilización y de 2.22\% para niveles de fertilización, aceptable para este tipo de experimentos. El promedio general del experimento fue de 37.4 hojas a las 40 semanas. 
Tukey al $5 \%$ para número de hojas a las 40 semanas, Cuadro 4, establece dos rangos de significancia para niveles de fertilización, ubicándose en el rango "a" los niveles n5 con 38.12 hojas, n3 y n1. En último lugar en el rango "b" está n2 (40 kg ha-1 N + 60 kg ha-1 P2O5 + 100 kg ha-1 K2O), con 36.32 hojas.

\begin{tabular}{|l|l|l|l|l|}
\hline \multirow{2}{*}{ F de V } & GL & \multicolumn{3}{|c|}{ Cuadrados Medios } \\
Total & & 20 semanas & 30 semanas & 40 semanas \\
Tipo de Fertilizac. & 15 & $0.18 \mathrm{~ns}$ & $14.185 \mathrm{~ns}$ & $14.76 \mathrm{~ns}$ \\
Error (a) & 2 & $3.06 \mathrm{~ns}$ & $0.78 \mathrm{~ns}$ & $1.10 \mathrm{~ns}$ \\
Niveles de Fertil. & 5 & $1.12 \mathrm{~ns}$ & 1.91 & 1.38 \\
t vs. n1,n2,n3,n4,n5 & 1 & $0.001 \mathrm{~ns}$ & $0.03 \mathrm{~ns}$ & $0.00 \mathrm{~ns}$ \\
$\mathrm{n} 5$ vs. $\mathrm{n} 1, \mathrm{n} 2, \mathrm{n} 3, \mathrm{n} 4$ & 1 & $0.006 \mathrm{~ns}$ & $0.03 \mathrm{~ns}$ & $0.11 \mathrm{~ns}$ \\
$\mathrm{n} 4$ vs. $\mathrm{n} 1, \mathrm{n} 2, \mathrm{n} 3$ & 1 & $0.021 \mathrm{~ns}$ & $0.01 \mathrm{~ns}$ & $0.00 \mathrm{~ns}$ \\
$\mathrm{n} 2$ vs. $\mathrm{n3}$ & 1 & $0.113 \mathrm{~ns}$ & $0.18 \mathrm{~ns}$ & $0.16 \mathrm{~ns}$ \\
$\mathrm{n} 4$ vs. $\mathrm{n} 5$ & 1 & $0.001 \mathrm{~ns}$ & $0.005 \mathrm{~ns}$ & $0.07 \mathrm{~ns}$ \\
Tx N & 5 & $0.83 \mathrm{~ns}$ & $0.0735 \mathrm{~ns}$ & $0.227 \mathrm{~ns}$ \\
Error (b) & 20 & 0.51 & 0.49 & 0.69 \\
CV(a) & & $10.45 \%$ & $5.81 \%$ & $3.14 \%$ \\
CV(b) & & $4.28 \%$ & $2.95 \%$ & $2.22 \%$ \\
\hline
\end{tabular}

Cuadro 4. Promedios y pruebas de significancia para número de hojas en el estudio de tipos y niveles de fertilización en plátano (Mussa AAB). El Carmen. Manabí. 2007.

\begin{tabular}{|c|c|c|c|c|}
\hline Factores & & \multicolumn{3}{|c|}{ Promedios (hojas/planta) } \\
\hline Tipos de Fertilizante & Significado & 20 semanas & 30 semanas & 40 semanas \\
\hline $\mathrm{T} 1$ & Fertiriego & 16.97 & 23.93 & 37.54 \\
\hline $\mathrm{T} 2$ & Fertilic. sólida & 16.54 & 23.63 & 37.19 \\
\hline Niveles de Fertilizante & & & & \\
\hline $\mathrm{N} 1$ & $20-30-50$ & 16.88 & $23.87 a$ & $37.63 a$ \\
\hline N2 & $40-60-100$ & 15.92 & $22.87 \mathrm{~b}$ & $36.32 \mathrm{~b}$ \\
\hline N3 & $60-90-150$ & 17.08 & $24.33 a$ & $37.72 a$ \\
\hline N4 & $40-60-100-26-13$ & 17.03 & $23.98 a$ & $37.18 a b$ \\
\hline N5 & $60-90-150-52-26$ & 16.93 & $24.23 a$ & $38.12 \mathrm{a}$ \\
\hline N6 testigo 16.68 & 23.40ab 37.25ab & & & \\
\hline $\mathrm{N} \times \mathrm{T}$ & & & & \\
\hline $\mathrm{t} 1 \mathrm{n} 1$ & & 17.40 & 24.30 & 37.83 \\
\hline $\mathrm{t} 1 \mathrm{n} 2$ & & 16.40 & 23.27 & 36.57 \\
\hline $\mathrm{t} 1 \mathrm{n} 3$ & & 17.33 & 24.63 & 37.87 \\
\hline $\mathrm{t} 1 \mathrm{n} 4$ & & 16.63 & 23.47 & 37.03 \\
\hline t1n5 & & 17.43 & 24.33 & 38.57 \\
\hline testigo & & 16.63 & 23.57 & 37.40 \\
\hline $\mathrm{t} 2 \mathrm{n} 1$ & & 16.37 & 23.43 & 37.43 \\
\hline $\mathrm{t} 2 \mathrm{n} 2$ & & 15.43 & 22.47 & 36.07 \\
\hline $\mathrm{t} 2 \mathrm{n} 3$ & & 16.83 & 24.03 & 37.57 \\
\hline $\mathrm{t} 2 \mathrm{n} 4$ & & 17.43 & 24.50 & 37.33 \\
\hline t2n5 & & 16.43 & 24.13 & 37.67 \\
\hline testigo & & 16.73 & 23.23 & 37.10 \\
\hline
\end{tabular}

Peso del racimo (Peso Fresco)

Al realizar el análisis estadístico para la variable peso del racimo Cuadro 5, se observa diferencias altamente significativas para niveles de fertilización y para la interacción
T x N. El coeficiente de variación fue de 3.17\% para tipo de fertilización y de 2.24\% para niveles de fertilización. El promedio general para esta variable fue de $11.44 \mathrm{~kg}$ por racimo. 
En el Cuadro 6, se aprecia que existen diferencias matemáticas para tipos de fertilización, ubicándose en primer lugar t1 (fertiriego) con 11.67kg y en último lugar t2 (fertilización edáfica) con $11.21 \mathrm{~kg}$. Tukey al 5\% para peso del racimo, Cuadro 8, para niveles de fertilización establece cuatro rangos de significancia, ubicándose en el rango "a" el nivel n5 (60 kg ha-1 N + 90 kg ha-1 P2O5 + 150 kg ha- $1 \mathrm{~K} 2 \mathrm{O}+52 \mathrm{MgO}+26$ SO3), con $12.06 \mathrm{~kg}$ por racimo y en último lugar n4 (40 kg ha-1 N + 60 kg ha-1 P2O5 + 100 kg ha-1 K2O + $26 \mathrm{MgO}+13$ SO3), con $11.01 \mathrm{~kg}$. Para la interacción T x N se observa sies rangos de significancia, en primero lugar se ubicó t1 n5 (fertiriego + (60 kg ha-1 N + 90 kg ha-1 P2O5 + 150 kg ha-1 K2O + $52 \mathrm{MgO}+26 \mathrm{SO} 3)$, con $12.35 \mathrm{~kg}$ y en último lugar t2n3 (fertilización edáfica + 60 kg ha-1 N + 90 kg ha-1 P2O5 + 150 kg ha-1 K2O), con $10.74 \mathrm{~kg}$ por racimo

\section{Materia Seca por Racimo}

Al realizar el análisis estadístico para la variable materia seca por racimo Cuadro 5, se observa diferencias significativas para tipo de fertilización y altamente significativas para niveles de fertilización y para la interacción $\mathrm{T}$ x N . El coeficiente de variación fue de $2.34 \%$ para tipo de fertilización y de 1.90\% para niveles de fertilización. El promedio general para esta variable fue de $3.28 \mathrm{~kg}$ por racimo.

Cuadro 5. Adeva para peso fresco del racimo y materias seca en plátano (Mussa AAB). El Carmen. Manabí. 2007

\begin{tabular}{|c|c|c|c|}
\hline F de V & $\mathrm{GL}$ & \multicolumn{2}{|c|}{ Cuadrados Medios } \\
\hline & & Peso fresco & Materia Seca \\
\hline Total & 35 & & \\
\hline Tipo de Fertilizac. & 1 & $1.891 \mathrm{~ns}$ & $0.162^{*}$ \\
\hline Error (a) & 2 & 0.131 & 0.006 \\
\hline Niveles de Fertil. & 5 & $0.849^{* *}$ & $0.083^{* *}$ \\
\hline t vs. $n 1, n 2, n 3, n 4, n 5$ & 1 & $0.027 \mathrm{~ns}$ & $0.0001 \mathrm{~ns}$ \\
\hline $\mathrm{n} 5$ vs. $\mathrm{n} 1, \mathrm{n} 2, \mathrm{n} 3, \mathrm{n} 4$ & 1 & $3.053^{* *}$ & $0.002 \mathrm{~ns}$ \\
\hline $\mathrm{n} 4$ vs. $\mathrm{n} 1, \mathrm{n} 2, \mathrm{n} 3$ & 1 & $0.524^{*}$ & $0.165^{* *}$ \\
\hline $\mathrm{n} 2$ vs. $\mathrm{n} 3$ & 1 & $0.545^{* *}$ & $0.236^{* *}$ \\
\hline n4 vs. n5 & 1 & $0.099 \mathrm{~ns}$ & $0.009 \mathrm{~ns}$ \\
\hline $\mathrm{T} \times \mathrm{N}$ & 5 & $0.339 * *$ & $0.0022^{* *}$ \\
\hline Error (b) & 20 & 0.065 & 0.004 \\
\hline CV(a) & & $3.17 \%$ & $2.34 \%$ \\
\hline$C V(b)$ & & $2.24 \%$ & $1.90 \%$ \\
\hline
\end{tabular}

Cuadro 6. Promedios y pruebas de significancia para número de hojas en el estudio de tipos y niveles de fertilización en plátano (Mussa AAB). El Carmen. Manabí. 2007.

\begin{tabular}{|l|l|l|l|}
\hline Factores & & \multicolumn{2}{|c|}{ Promedios } \\
Tipos de Fertilizante & Significado & Peso fresco & Materia Seca \\
T1 & Fertiriego & 11.67 & $3.34 \mathrm{a}$ \\
T2 & Fertilic. sólida & 11.21 & $3.21 \mathrm{~b}$ \\
Niveles de Ferti. & & & \\
N1 & $20-30-50$ & $11.57 \mathrm{~b}$ & $3.43 \mathrm{a}$ \\
N2 & $40-60-100$ & $11.38 \mathrm{~b}$ & $3.37 \mathrm{ab}$ \\
N3 & $60-90-150$ & $11.11 \mathrm{c}$ & $3.16 \mathrm{~d}$ \\
N4 & $40-60-100-26-13$ & $11.01 \mathrm{~cd}$ & $3.13 \mathrm{~d}$ \\
N5 & $60-90-150-52-26$ & $12.06 \mathrm{a}$ & $3.29 \mathrm{bc}$ \\
N6 & testigo & $11.50 \mathrm{~b}$ & $3.28 \mathrm{c}$ \\
& N $\mathrm{xT}$ & Peso fresco & Materia Seca \\
& t1n1 & $12.14 \mathrm{a}$ & $3.49 \mathrm{a}$ \\
& t1n2 & $11.28 \mathrm{de}$ & $3.42 \mathrm{ab}$ \\
& t1n3 & $11.47 \mathrm{bcd}$ & $3.25 \mathrm{~d}$ \\
& t1n4 & $11.07 \mathrm{ef}$ & $3.14 \mathrm{e}$ \\
& t1n5 & $12.35 \mathrm{a}$ & $3.47 \mathrm{a}$ \\
& testigo & $11.69 \mathrm{bc}$ & $3.30 \mathrm{~cd}$ \\
& t2n1 & $10.99 \mathrm{bc}$ & $3.37 \mathrm{bc}$ \\
& t2n2 & $11.49 \mathrm{ef}$ & $3.33 \mathrm{bcd}$ \\
& t2n3 & $10.74 \mathrm{bcd}$ & $3.06 \mathrm{e}$ \\
& t2n4 & $10.95 \mathrm{ef}$ & $3.12 \mathrm{e}$ \\
& t2n5 & $11.77 \mathrm{~b}$ & $3.12 \mathrm{e}$ \\
& testigo & $11.31 \mathrm{cde}$ & $3.26 \mathrm{~d}$ \\
\hline
\end{tabular}


DMS al 5\% para tipos de fertilización, Cuadro 6, establece dos rangos de significancia, ubicándose en primer lugar t1 (fertiriego) con $3.34 \mathrm{~kg}$ por racimo de materia seca, y en último lugar a t2 (fertilización edáfica), con $3.21 \mathrm{~kg}$. Tukey al 5\% Cuadro 8, para niveles de fertilización, establece cuatro rangos de significancia, en primer lugar se ubicó el nivel n1 (20 kg ha-1 N + 30 kg ha-1 P2O5 + 50 kg ha-1 K2O), con 3.43kg y en último lugar n4 (40 kg ha-1 N + 60 kg ha-1 P2O5 + 100 kg ha-1 $\mathrm{K} 2 \mathrm{O}+26 \mathrm{MgO}+13 \mathrm{SO}$ ), con $3.13 \mathrm{~kg}$. Para la interacción $\mathrm{T} \times \mathrm{N}$ se encontró cinco rangos de significancia, ubicándose en el primer rango las interacciones con fertiriego t1n1 con $3.49 \mathrm{~kg}$ y t $1 \mathrm{n} 5$ con $3.47 \mathrm{~kg}$ y en último lugar $\mathrm{t} 2 \mathrm{n} 3 \mathrm{con} 3.06 \mathrm{~kg}$ con fertilización edáfica.
$52 \mathrm{MgO}+26 \mathrm{SO} 3)$, con $12.01 \mathrm{~kg}$ por racimo. Sin embargo, n1 (20 kg ha-1 N + 30 kg ha-1 P2O5 + 50 kg ha-1 K2O) presentó el promedio más alto para peso en materia seca con $3.43 \mathrm{~kg} /$ racimo.

Para la interacción tipos de fertilización por niveles de fertilización, la mejor respuesta se presentó en t1n4 fertiriego + (40 kg ha-1 N + 60 kg ha-1 P2O5 + 100 kg ha-1 K2O + 26 kg ha-1 MgO + 13 kg ha-1 SO3), y t2n5 fertilización sólida + 60 kg ha-1 N + 90 kg ha-1 P2O5 + 150 kg ha-1 $\mathrm{K} 2 \mathrm{O}+52 \mathrm{MgO}+26$ SO3), con $2.3 \mathrm{~m}$ en altura de planta a las 30 semanas, mientras que a las 40 semanas el mejor promedio se presentó para t1n5 fertiriego + (60 kg ha-1 N + 90 kg ha-1 P2O5 + 150 kg ha-1 K2O $+52 \mathrm{MgO}+26 \mathrm{SO} 3) \mathrm{con} 4.2 \mathrm{~m}$. Tanto para peso fresco como

Cuadro 7. Análisis Económico de Beneficio costo en el estudio de tipos y niveles de fertilización en plátano (Mussa AAB). El Carmen. Manabí. 2007.

\begin{tabular}{|c|c|c|c|c|}
\hline Interacción & Descripción & $\begin{array}{l}\text { Beneficio B. } \\
\text { USD/ha }\end{array}$ & $\begin{array}{l}\text { Costos Variables } \\
\text { Total (USD/ha) }\end{array}$ & $\begin{array}{l}\text { Relación } \\
\text { B/C }\end{array}$ \\
\hline testigo & & 6867.19 & 845.40 & 8.12 \\
\hline testigo & & 6645.82 & 845.40 & 7.86 \\
\hline $\operatorname{t1n} 1$ & Fertiriego, 20-30-50 & 7131.58 & 971.40 & 7.34 \\
\hline $\mathrm{t} 2 \mathrm{n} 1$ & Fertilización sólida, 20-30-50 & 6459.75 & 905.84 & 7.13 \\
\hline $\mathrm{t} 2 \mathrm{n} 2$ & Fertilización sólida, 40-60-100 & 6751.58 & 966.29 & 6.99 \\
\hline $\mathrm{t} 2 \mathrm{n} 4$ & Fertilización sólida, & & & \\
\hline \multirow{3}{*}{$\mathrm{t} 2 \mathrm{n} 5$} & $40-60-100-26-13$ & 6432.36 & 991.07 & 6.49 \\
\hline & Fertilización sólida, & & & \\
\hline & $60-90-150-52-26$ & 6918.07 & 1076.29 & 6.43 \\
\hline $\operatorname{t} 1 \mathrm{n} 2$ & Fertiriego, 40-60-100 & 6626.24 & 1071.17 & 6.19 \\
\hline $\mathrm{t} 2 \mathrm{n} 3$ & Fertilización sólida, 60-90-150 & 6308.94 & 1026.73 & 6.14 \\
\hline $\mathrm{t} 1 \mathrm{n} 4$ & Fertiriego, 40-60-100-26-13 & 6506.78 & 1129.22 & 5.76 \\
\hline $\operatorname{t1n} 3$ & Fertiriego, 60-90-150 & 6741.79 & 1170.93 & 5.76 \\
\hline $\mathrm{t} 1 \mathrm{n} 5$ & Fertiriego, 60-90-150-52-26 & 7258.90 & 1287.03 & 5.64 \\
\hline
\end{tabular}

De la relación beneficio-costo Cuadro 7, se concluye que el tratamiento testigo (sin fertilización), reporta el mayor beneficio-costo con 8.12, lo que significa que por cada dólar invertido, recibirá 8.12 dólares de ganancia. La comercialización se realizó en cajas $22 \mathrm{XU}$ para exportación con un peso de $23 \mathrm{~kg}$ de fruta.

\section{Conclusiones}

Para tipo de fertilización se presentaron únicamente diferencias matemáticas a favor de fertiriego para número de hojas, altura de planta y peso fresco. Para peso de materia seca, el mejor tratamiento con significancia estadística fue t1 (fertiriego), con $3.34 \mathrm{~kg}$ por racimo de plátano.

El nivel de fertilización que presentó los mejores promedios para altura de planta, número de hojas y peso fresco fue n5 (60 kg ha-1 N + 90 kg ha-1 P2O5 + 150 kg ha-1 K2O + para materia seca, las mejor interacción fue t1n5 fertiriego + (60 kg ha-1 N + 90 kg ha-1 P2O5 + 150 kg ha-1 K2O + 52 MgO + 26 SO3) y t1n1 fertiriego + (20 kg ha-1 N + 30 kg ha-1 P2O5 $+50 \mathrm{~kg}$ ha-1 K2O), 12.35 y $12.14 \mathrm{~kg}$ por racimo en fresco y 3.49 y $3.47 \mathrm{~kg}$ en seco.

En el análisis económico se observó que la mejor interacción fue el testigo (sin fertilización), tanto el que se ubicó en la parcela de fertiriego como el de fertilización sólida. Presentaron la más alta relación beneficio costo con 8.12 y 7.16. Belalcázar (1991), sostiene que el cultivo de plátano se aparta de cualquier clase lógica fundamentada por el hecho de que la mayoría de los suelos aptos para su cultivo, con muy pocas excepciones, disponen de nutrimentos en las cantidades requeridas para producir como mínimo un primer ciclo de cosecha, por lo tanto cualquier cantidad extra que se le aplique no va a reportar ningún beneficio económico debido a que no hay ningún incremento en la producción o si la hay, 
ésta no alcanza a compensar la inversión correspondiente a dicho factor de producción. Sin embargo, se esperaba en este ensayo que por el uso del riego, exista una respuesta favorable del cultivo a la aplicación de fertilizantes, que a la final, no fue suficiente.

\section{Recomendaciones}

En las condiciones en las que se desarrolló el ensayo, es decir, un suelo con fertilidad media, alto contenido de materia orgánica y con textura franco limosa, la recomendación sería no fertilizar el cultivo, al menos en el primer ciclo de producción.

Evaluar la respuesta del cultivo de plátano a la fertilización, en dos ciclos continuos de producción. Ya que es muy probable que la riqueza natural del suelo en nutrientes solo sea suficiente para suplir adecuadamente un ciclo de producción.

\section{Bibliografía}

- BelalCAZAR, S. (1991). El Cultivo de Plátano (Musa AAB Simmonds) en el Trópico. Instituto Colombiano Agropecuario (ICA). Red Internacional para el mejoramiento del banano y plátano. INIBAP - LAC. Manual de asistencia técnica No 50. pp 103-239. Colombia.

- BERTSCH, F. (2003). Absorción de Nutrimentos por los Cultivos. Universidad de Costa Rica. CIA. Asociación Costarricense de la Ciencia del Suelo (ACCS). San José (Costa Rica). pp 248-250.

- CARRANZA, M. (1999). Evaluación de la Densidad de Plantación utilizando doble hilera en el primer ciclo de producción del cultivo de plátano (Musa AAB), var. Barraganete enano. Santo Domingo de los Tsachilas. Tesis de Grado. Universidad Central Del Ecuador. Quito (Ecuador).

- CENTA. (2002). Guía Técnica del Cultivo de Plátano. Centro Nacional de Tecnología Agropecuaria y Forestal. La Libertad (El Salvador).

- ORELLANA J., UNDA J., y ANALUISA P. (2002). Estudio de Comercialización del Plátano en la Zona Norte del trópico Ecuatoriano. Publicaciones Misceláneas № 113. Ecuador.

• PEÑA W. (2003). Fertilización con Nitrógeno, Fósforo y Potasio en Plantaciones establecidas de plátano barraganete (Musa paradisiaca L), en la zona de El Carmen. Tesis. Ing. Agrómono. Universidad Laica Eloy Alfaro. ULEAN. Manabí. Ecuador.

- SICA. (2001). Identificación de Mercados y Tecnología para Productos Agrícolas Tradicionales de Exportación. Plátano Musa spp. Consultado Abril 2005. pp3-11. disponible en: www.sica. gov.ec/agronegocios

- SUAREZ A. (1992). Recomendación de Fertilización en Plátano. In Manual de Producción del Plátano. Fundación hondureña de
Investigación Agrícola (FHIA). pp 63-71.

- TOAPANTA J., (2004). Efecto de la Fertilización y Altas Densidades de Plantas Sobre el Rendimiento del cultivo de Plátano, en la zona de Quevedo. Sociedad Ecuatoriana de la Ciencia del Suelo (SECS). IX Congreso Ecuatoriano de la Ciencia del Suelo. Loja. Ecuador.

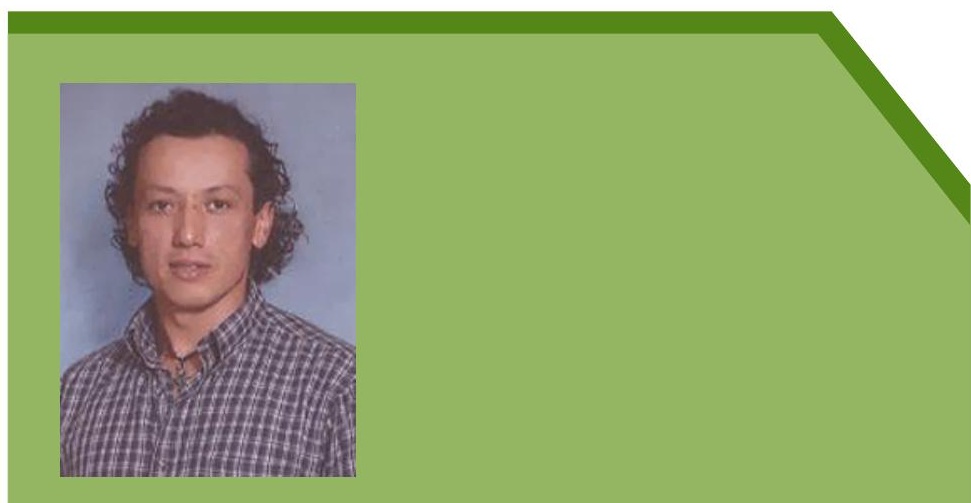

Autor. Diego Alfonso Vaca Sotelo

Egresado de la Maestría en Nutrición Vegetal de la Universidad Tecnológica Equinoccial, Ingeniero Agrónomo de la Universidad Central del Ecuador.

Actualmente es administrador de la Finca Santa Marianita en El Carmen - Manabí. Ha desempeñado cargos como Gerente Comercial de Agropacífico, Interandesa S.A, y fue Promotor de AFECOR Febres Cordero CIA. LTDA.

Ha realizado cursos y seminarios en cultivo de babaco bajo invernadero, agroempresa y sostenibilidad, poricnotecnia, lombricultura, cultivo de rosas, entre otros.

Durante su vida estudiantil se ha destacado por un excelente rendimiento académico y deportivo. 